\title{
Modeling of chemical erosion of graphite due to hydrogen by inclusion of chemical reactions in SDTrimSP
}

\author{
A. Rai, A. Mutzke and R. Schneider
}

Max-Planck-Institut für Plasmaphysik, D-17491 Greifswald, Germany

Corresponding Author: Abha Rai

e-mail: Abha.Rai@ipp.mpg.de

Phone: +49 3834 882400, Fax: +49 3834882409

Postal Address: Max-Planck-Institut für Plasmaphysik, D-17491 Greifswald, Wendelsteinstrasse 1, Germany 
Abstract: Erosion of carbon based materials plays a very crucial role in determining its candidature as a potential plasma facing material in fusion devices. Carbon erosion yield shows strong dependence on energy, flux of the incoming ions and the target temperature. Various experiments and theoretical models have made successful attempts to understand some aspects of the erosion mechanism of carbon bombarded by hydrogen ions. They are valid for a particular parameter range. None of the models alone can explain the temperature, energy and flux dependence of erosion simultaneously. In the present work different aspects of erosion mechanism are parameterized. A new model which takes into account the basic merits of different theoretical models and the various parameterized quantities is embedded in the SDTrimSP [1,2] program. This upgraded SDTrimSP enables us to calculate the physical and chemical sputtering in a single model for various parameter ranges. The results obtained by the upgraded SDTrimSP are in good agreement with experimental data.

Key words: Hydrogen; TRIM; Chemical erosion; Carbon; Physical sputtering; Diffusion

\section{Introduction}

Erosion of carbon-based materials due to particle impact has long been a concern when it comes to plasma-facing applications in thermonuclear fusion reactors. Although these materials exhibit excellent thermo-mechanical properties, their susceptibility to erosion through physical and chemical processes limits their lifetimes while increasing plasma impurity levels. In particular, there is large uncertainty about the extrapolation of the chemical sputtering yield to fusion reactor relevant fluxes.

The chemical reaction of energetic ions with carbon atoms occurs after slowing down of the ions. Three main mechanisms have been identified for the chemical erosion

(1) Thermally activated process occurring due to the local chemistry of reactions between $\mathrm{H}$ and $\mathrm{C}$

(2) Radiation damage occurring due to the energy deposition by the energetic $\mathrm{H}$ atoms

(3) Kinetic hydrocarbon emission or surface effects, e. g. swift chemical sputtering. This contribution dominates the chemical erosion at low energies.

Different models for understanding the chemical erosion and its dependence on temperature, energy and flux are existing [3-7]. Most of these models are based on the Küppers cycle $[4,5]$ of chemical erosion. They are able to reproduce 
the available experimental results for specific parameter ranges. The model of Mech [3] gives the most complete atomistic picture of chemical erosion. Since the Küppers cycle takes into account only the contribution from the thermally activated processes the model was extended to include the other contributions to chemical erosion namely radiation damage and surface kinetic hydrocarbon emission in the atomistic frame work. The goal of the present work is to include a chemistry-based module for hydrocarbon reactions into the collisional cascade SDTrimSP version 5.0 to model the experimental results of the energy, temperature and flux dependence of chemical erosion (i) due to hydrogen bombardment and (ii) due to combined $A r^{+}$and $\mathrm{H}$ bombardment. In the following section most of the existing models of chemical erosion are discussed briefly.

\subsection{Model by Roth}

Roth et al. [6] proposed an analytical model for the chemical erosion of carbon by hydrogen ion bombardment. The total erosion yield of carbon under hydrogen bombardment, $Y_{\text {tot }}$, consists of three components, the physical sputtering yield $\left(Y_{\text {phys }}\right)$, the thermal erosion yield $\left(Y_{\text {therm }}\right)$, enhanced by radiation damage $\left(Y_{\text {dam }}\right)$ and the sputtering of radicals $\left(Y_{\text {surf }}\right)$, see Appendix A.

$$
Y_{\text {tot }}=Y_{\text {phys }}+Y_{\text {therm }}\left(1+D \cdot Y_{\text {dam }}\right)+Y_{\text {surf }}
$$

In general, the predicted yields shows good agreement with the experimentally observed values. But in this model, as argued by Mech et al., purely empirical forms of the equations are chosen rather than allowing the individual reactions to proceed within the atomistic framework of the model system.

\subsection{Model by Hopf}

Hopf et al. [7] proposed that the bombardment of a carbon target by $A r^{+}$ ions in the presence of atomic hydrogen leads to broken bonds created due to the collision cascade in the target. They are instantly passivated by the abundant flux of the atomic hydrogen, which penetrate a few nanometers into the target. This process leads to the formation of stable hydrocarbons within the overlapping range of $\mathrm{Ar}^{+}$ions and H. Formed hydrocarbon molecules diffuse to the surface and finally gets desorbed. The proposed mechanism gives an explanation to the experimentally observed time constant $(\sim 1 \mathrm{~ms})$ as the time needed for out-diffusion of the erosion products. As a consequence of this, at very high hydrogen fluxes this out-diffusion becomes the rate limiting step for chemical sputtering. 
On the basis of the above proposed mechanism of chemical erosion, Hopf et al. have developed a simple model. The energy dependence of the chemical sputtering yield is proportional (i) to the yield $Y_{c}$ at which the incoming ion breaks $C-C$ bonds and (ii) to the probability $p_{\text {pass }}$ for the passivation of a broken bond by atomic hydrogen. The maximum range of diffusion, $d$, of atomic hydrogen in carbon and in $\mathrm{C}: \mathrm{H}$ films was determined to be about 2 nm. $p_{\text {pass }}$ is assumed to be an exponential decaying function of the distance to the surface, with a mean free path of $\lambda$. The total erosion yield is given by

$$
\begin{aligned}
& Y_{\text {tot }}=Y_{\text {phys }}+Y_{\text {chem }} \\
& Y_{\text {chem }}=a \cdot \int_{0}^{d} Y_{c} \cdot p_{\text {pass }} d x \\
& Y_{\text {chem }}=a \cdot \int_{0}^{d} Y_{c} \cdot e^{(-x / \lambda)} d x
\end{aligned}
$$

Here, $a=0.4, \lambda=0.4 \mathrm{~nm}$ and $d=2 \mathrm{~nm}$.

\subsection{Extended Küppers model by Mech}

Küppers and co-workers $[4,5]$ outlined the principal atomistic processes involved in the chemical erosion of amorphous hydrogenated carbon (a-C:H) by the impact of thermal hydrogen atoms. This model is appropriate to study the interaction of hydrogen with partially or fully hydrogenated carbon materials and was later extended by Mech et al. to develop a self consistent energetic model for chemical erosion. It is well known that the interaction of energetic $\mathrm{H}$ with $\mathrm{C}$ leads to a- $\mathrm{C}: \mathrm{H}$ and the resulting sample has a large internal surface area which plays a very important role in the hydrocarbon formation. Mech et al. have used this "effective surface" approach to implement this. Since this effective surface depends on the ion impact energy, they take the total flux density as used in the experiment and use some fitting parameters (described in the following paragraph) to implement this aspect.

Fig. 1 shows various atomistic processes occurring due to the impact of the thermal H-atom on amorphous hydrogenated carbon (a-C:H) films [8]. For the detailed set of rate equations the reader is referred to Appendix B. The methane erosion yield is composed of a kinetic part $Y_{0}$ and a thermal part $Y_{T}$. For given steady state concentration of $s p^{3}$ and $s p_{C H}^{x}$ and $\sigma_{i}$ the methane yield is:

$$
Y_{\mathrm{CH}_{4}}=\quad Y_{0}\left(E, T_{0}\right) \quad+Y_{T}(E, T)
$$




$$
\begin{aligned}
& Y_{C H_{4}}=\varrho \cdot \sigma_{i} \cdot\left[s p^{3}+s p_{C H}^{x}\right]+\varrho \cdot s p_{C H}^{x} \frac{E x}{\Phi} \\
& Y_{\mathrm{CH}_{4}}=\quad Y_{\text {OMech }}+Y_{\text {TMech }}
\end{aligned}
$$

$Y_{0 M e c h}\left(=\varrho \cdot \sigma_{i} \cdot\left[s p^{3}+s p_{C H}^{x}\right]\right)$ and $Y_{T M e c h}\left(=\varrho \cdot s p_{C H}^{x} \frac{E x}{\Phi}\right)$ are the kinetic and thermal parts of the erosion yields respectively obtained from the Mech model. $\varrho$ is the surface density of carbon atoms: $\varrho=6.0 \cdot 10^{19}$ atoms $/ \mathrm{m}^{2}$. The bracketed quantities represent concentrations and $\Phi$ the $\mathrm{H}$ atom flux.

The steady-state solution of the above presented set of differential equations reproduces the experimental results of the temperature and energy dependence of chemical erosion very well for a flux of $10^{18} \mathrm{~m}^{-2} \mathrm{~s}^{-1}$. Since the fitting parameters were calculated for this particular flux value, the results obtained by this model for the other fluxes are far from the experimental data. The flux dependence of the erosion yield predicted from the above model is also too strong.

\subsection{Model by Salonen et al.}

An attempt was made by Salonen et al. $[9,10]$ to explain the flux dependence of chemical erosion using Molecular Dynamics (MD). An a:C-H sample was bombarded with hydrogen ions of $10 \mathrm{eV}$ energy and at a rate of $2 \times 10^{29}$ ions $/ \mathrm{m}^{2} \mathrm{~s}$. The hydrogen content of the surface first increased strongly and after reaching a $\mathrm{H} / \mathrm{C}$ ratio of about 0.56 the sample became super-saturated. After this the hydrogen content in the sample increased only slightly, as the surface was already saturated. The erosion yield for the unsaturated surface was $\sim 0.01$, while for the super-saturated surface it was only $\sim 0.001$. The drop of one order of magnitude in the observed erosion yield was believed to be the explanation to the experimentally observed decrease in the erosion yield. The reason to the sharp drop was the decreased carbon collision cross section at a surface which had obtained the temporary super-saturation of $\mathrm{H}$ atoms due to the extremely high flux involved. The flux value considered here is very high compared to the fusion relevant fluxes $\left(10^{24}\right.$ ions $\left./ \mathrm{m}^{2} / \mathrm{s}\right)$.

\subsection{Model by Rai et al.}

Inspired by the work of Salonen et al., another attempt was made by Rai et al. [11] to study the flux dependence of chemical erosion. The Küppers cycle of chemical erosion was implemented in a 3D multi-scale model to simulate the hydrogen reactive-diffusive transport in porous graphite [12]. The basic prin- 
ciple behind the 3D multi-scale model was to use the insights gained from the microscopic models (using MD or ab-initio methods) for modeling the transport at the meso-scale (using Kinetic Monte Carlo (KMC) method). Then the results of the chemical erosion yield obtained from the meso-scale were used as input to a simple geometrical model. The basic idea of the geometrical model was that due to the shielding for deeper lying carbon atoms, the origin of the total amount of carbon that could be released was limited to a few surface layers. The hydrocarbon molecules which were present in the deeper layers collided with the other target atoms before they reached the surface and got stuck. This idea is very similar to the model of Hopf et al.. This geometrical constraint for the release of hydrogen molecules created a strong flux dependence.

The local chemistry from the Küppers model was used to calculate the hydrocarbon molecule formation process and then the release probability of the produced hydrocarbon based on the purely geometrical constraints was determined numerically. The Chemical erosion yield obtained from the 3D KMC model, $Y_{C h e m}$, gives the number of $\mathrm{CH}_{3}$ molecules produced per incident hydrogen atom from the sample with given internal structure, at a given temperature and ion energy. Then a ray tracing kind of technique was used in the 3D spherical volume to find $P_{\text {Geometry }}$ (release probability of the hit hydrocarbon molecule on the basis of geometrical constraints). $P_{\text {Geometry }}$ was calculated from the ratio of the number of hits with other carbon atoms (lying more shallowly than the chosen carbon atom) to the total number of trials. One starts with an ordered graphite structure, and, after the hydrogen ions hit the carbon atoms, the structure of the sample is updated. The release probability calculated by this method is due to the geometry of the sample and the location of the particular carbon atom hit by the hydrogen atom. The actual release probability $\left(P_{\text {Release }}\right)$ is

$$
P_{\text {Release }}=P_{\text {Geometry }} \times Y_{\text {Chem }}
$$

So the quantity $P_{\text {Release }}$, includes the effect of the chemistry processes proposed by Küppers as well as the effect of the geometrical constraints on the carbon release probability due to the geometrical structure of the sample and the position of the hit carbon atom.

The yield obtained by the KMC model itself shows very weak flux dependence but the combination of KMC with the simple geometrical model gives quite encouraging results. The obtained flux dependence is in very good agreement with the experimental results for a wide range of fluxes. The strong flux dependence predicted by the geometrical model is consequence of the nearly constant carbon flux due to the depth limit of the released carbon from the geometrical blocking effect. The only drawback of this model is that the KMC model includes only thermally activated processes and the other two contribu- 
tions coming from the momentum transfer $i$. e. energy deposition and kinetic hydrocarbon emission can not be included and therefore the picture is not complete. However, since the time scale of the binary collision processes and the diffusion related processes (depending on target temperature) in KMC are by $10^{2}-10^{5}$ orders of magnitude different, this is not possible.

\subsection{Summary of existing models}

Fig. 2 shows the experimental data for the energy dependence of methane erosion yield of a carbon sample at room temperature bombarded by energetic hydrogen ions. The simulation results obtained by Mech, Roth, and Hopf are also shown for comparison. It is evident from the figure that the model of Hopf is far from the experimental values. The curves from Mech and Roth shows good agreement.

Fig. 3a shows the sputtering yields of energetic Ar bombardment on an a-C:H film at 300K. Fig. 3b shows the experimental data for the same experiment but with the presence of thermal atomic hydrogen. If one compares the two figures it can be noted that for the case of Ar bombardment in the presence of thermal hydrogen the sputtering yield was much higher. The reason is that in the second experiment thermal hydrogen plays an important role in chemical erosion which eventually leads to higher sputtering yields (physical sputtering + chemical sputtering). The models from Mech and Roth are not able to reproduce this effect, whereas the results calculated using Hopf are in good agreement with the experiments.

Following the observations one can conclude that the model of Hopf can successfully explain the results of $A r^{+} \mid H \rightarrow C$ but not $H^{+} \rightarrow C$ (Figs. 2-3). The model of Roth and Mech can explain $H^{+} \rightarrow C$ but not $A r^{+} \mid H \rightarrow C$.

Fig. 4 shows the temperature dependence of the erosion yield for $200 \mathrm{eV}$ and Fig. 5 shows the energy dependence of the erosion yield at $700 \mathrm{~K}$. It can be seen that the results obtained by Mech matches very well with the experiments. Whereas the model of Hopf is far off the experimental values.

Figs. 6 and 7 shows the flux dependence of the erosion yield at $700 \mathrm{~K}$ and 800 $\mathrm{K}$ from [13]. The flux dependence calculated from the model of Hopf and Mech are far off the experimental data. Only the results from the analytical model of Roth [6] for chemical erosion show correct tendencies of the flux dependence. From the survey of Figs. 2-7 it is clear that none of the analytical models available predicts simultaneously the flux, temperature and energy dependence of erosion correctly. 


\section{Description of the new chemistry module}

In the present work, elements from the following ingredients were used to parameterize different aspects of chemical erosion and then included in the collisional cascade code SDTrimSP version 5.0 to model various experimental results.

(1) Binary collision SDTrimSP code: main code which simulates the interaction of energetic particles (e.g. H) with a target (e.g. C) taking into account also the changes within the target due to the bombardment and calculates various physical quantities like sputtering yield.

(2) Mech model for the chemical erosion: used as a subroutine in SDTrimSP code to handle the local chemistry and to cross-check the system evolution in terms of interchange of carbon centers with different hybridization states.

(3) Depth dependent escape probability: $\exp (-x / \lambda)$ gives the out-diffusion probability of a hydrocarbon molecule formed at depth $\mathrm{x}$.

In accordance to Eq. (B.12), the total methane erosion yield $\left(Y_{\mathrm{CH}_{4}}\right)$ in the new SDTrimSP code is calculated as a sum of a kinetic contribution ( $Y_{0 \_ \text {SDTrim }}$ at $T_{0}=300 \mathrm{~K}$ ) and of a temperature dependent or thermal contribution $\left(Y_{T \_S D T r i m}\right)$ :

$$
Y_{C H_{4}}=Y_{0 \_S D T r i m}\left(E, T_{0}\right)+Y_{T \_S D T r i m}(E, T)
$$

First, the carbon species included in the new model for chemistry will be discussed and then the procedure to calculate $Y_{0 \_ \text {SDTrim }}\left(E, T_{0}\right)$ and $Y_{T_{-} \text {SDTrim }}(E, T)$ will be presented.

\subsection{Introduction of different carbon hybridization states}

In the original version of SDTrimSP carbon atoms were simulated as a single species. For simulating chemical erosion one needs to distinguish carbon atoms having different hybridization states as different species. Therefore, the new chemistry module describes an a- $\mathrm{C}: \mathrm{H}$ film in terms of three carbon species namely, $s p^{2}$ (carbon with double bonds), $s p^{3}$ (carbon with single bonds and not bonded to $\mathrm{H}$ atom) and $s p_{H}^{3}$ ( $s p^{3}$ carbon with a bonded $\mathrm{H}$ atom). The code has been modified to include the following chemical reactions:

(1) If an impinging ion transfers more than a critical value of energy to a $s p^{2}$ carbon then the double bond is broken and a $s p^{3}$ carbon is created. In the model of Hopf [7] the critical energy of $5.0 \mathrm{eV}$ was chosen. If this 
value is used in the code this gives negligible chemical erosion yield for the incident energy of $10 \mathrm{eV}$ in contrast to the experiments. A scan was done and it was found that a critical energy value of $3.6 \mathrm{eV}$ gives the best agreement with the experiments. This value is used in the present calculations.

(2) Since there is an upper limit of $1 / 3$ on $\mathrm{H}$ atoms bonded to $\mathrm{C}$ for typical $\mathrm{C}: \mathrm{H}$ film, this was used as a limit, $i . e$. ,

- if the concentration of $\mathrm{H}\left(n_{H}\right)$ or $s p^{3}\left(n_{s p^{3}}\right)$ exceeds the concentration of $s p_{H}^{3}\left(n_{s p_{H}^{3}}\right), s p^{3}$ carbon is changed to $s p_{H}^{3}$.

- if $n_{H}$ or $n_{s p^{3}}$ is less than $n_{s p_{H}^{3}}, s p_{H}^{3}$ is transferred to $s p^{3}$.

(3) The maximum concentration of carbon centers in $s p^{3}$ hybridization state $\left(s p^{3}, s p_{H}^{3}\right)$ is controlled (as a feedback mechanism) from the values calculated by the Mech model $\left(s p^{3}, s p_{H}^{3}, s p_{C H}^{3}\right)$ and is dependent on temperature. For example, at a given temperature, if the total concentration of carbon centers in $s p^{3}$ hybridization state calculated from SDTrimSP $\left(n_{s p^{3}}+n_{s p_{H}^{3}}\right)$ is greater than the corresponding value predicted by the Mech model, then $s p_{H}^{3}$ will change into $s p^{2}$ carbon.

Taking into account the reaction channels and the different carbon species presented above, the erosion yield is calculated as follows.

\subsection{Contribution from the kinetic part}

The calculation of the kinetic part, $Y_{0_{-} S D T r i m}\left(E, T_{0}\right)$, of the erosion yield is based on the mechanism proposed by Hopf [7]. When the low energy ions impinge on the carbon surface they cause bond breaking and finally get thermalized (implanted) within the sample. Lets say $Y_{B B}$ represents the number of bond breaking caused by an impinging ion and $Y_{H_{\text {stop }}}$ gives the yield of the $\mathrm{H}$ atom implantation. This stopped or implanted $\mathrm{H}$ atoms can be bonded to free open carbon bonds.

For hydrocarbon formation both open carbon bonds and hydrogen atoms must be available. For example in case of high energy bombardment of $\mathrm{H}$ on $\mathrm{C}$ a lot of open bonds are created near the surface but the hydrogen atom are eventually implanted much deeper in the sample where the number of broken $\mathrm{C}$ bonds is smaller and therefore the erosion yield at higher energies drops (see Fig. 3). In order to include this effect the minimum of $Y_{B B}$ and $Y_{H s t o p}$ is taken. For an out-diffusion probability of a hydrocarbon molecule from a depth $\mathrm{x}$ the kinetic part of the chemical erosion yield $Y_{0_{-} S D T r i m}\left(E, T_{0}\right)$ is given by

$$
Y_{0 \_ \text {SDTrim }}\left(E, T_{0}\right)=\int_{0}^{d} a \cdot \min \left[Y_{B B}, Y_{H_{\text {stop }}}\right] \cdot e^{(-x / \lambda)} d x
$$


Here, $\lambda(=0.4 \mathrm{~nm})$ is the typical range of out-diffusion for hydrocarbon and $\mathrm{d}$ is the depth of the sample. If the whole sample is divided into $\mathrm{k}$ strips or depth intervals parallel to the surface as in SDTrimSP

$$
Y_{0 \_S D T r i m}\left(E, T_{0}\right)=\sum_{0}^{k} a \cdot \min \left[Y_{B B}(k), Y_{H_{s t o p}}(k)\right] \cdot e^{(-x(k) / \lambda)} .
$$

The minimum condition $\min \left[Y_{B B}(k), Y_{H_{\text {stop }}}(k)\right]$ expresses the fact that chemical erosion only happens when sufficient open bonds $\left(Y_{B B}(k)\right)$ and sufficient hydrogen $\left(Y_{H_{s t o p}}(k)\right)$ are available. Therefore, the minimum of both will limit the production of hydrocarbons. The constant coefficient given by Hopf $(a=0.4)$ is replaced by a variable value ranging from 0.04 to 1.0 and was obtained by the comparison between measurements and simulation results.

$$
a=\min \left[1.0,0.04+0.01 \cdot \frac{Y_{H_{s t o p}}(k)}{Y_{B B}(k)}\right]
$$

The value of $a=1.0$ is found to reproduce quite well the measurements for hydrogen on carbon at low energies, whereas for high energies $a=0.04$ is needed. The linear parametrization is done as a function of $\frac{Y_{H_{s t o p}}(k)}{Y_{B B}(k)}$, because this ratio changes as a function of energy. Using this fit for the experiment of Hopf a slightly larger chemical erosion is obtained due to the larger values at small energies, but the data are still well described. 


\subsection{Contribution from the thermal part}

The temperature dependent part of the chemical erosion yield, $Y_{T \_S D T r i m}\left(E, T, \Phi_{0}\right)$, in the SDTrimSP code is based on the Mech model.

$$
Y_{T \_S D T r i m}\left(E, T, \Phi_{0}\right)=\sum_{1}^{n} \frac{Y_{T \_M e c h}\left(E, T, \Phi_{0}\right)}{s p^{3}+s p_{C H}^{x}} \cdot e^{(-x / \lambda)} .
$$

Here $\mathrm{n}$ is the total number of collisions between ions and $s p_{H}^{3}$. The depth dependent out-diffusion probability after the hydrocarbon formation $e^{(-x / \lambda)}$ is added. The term $Y_{T_{-} M e c h}\left(E, T, \Phi_{0}\right)$ (see Eqs. (B.12 - B.14)) is the yield of chemical erosion at a constant flux of $\left(\Phi_{0}=10^{18} \mathrm{~m}^{-2} \mathrm{~s}^{-1}\right)$.

\subsection{Flux dependence of chemical erosion}

It is observed that the kinetic part of the erosion yield is flux independent, therefore, in order to implement the correct flux dependence the thermal contribution $\left(Y_{\text {therm }}\right)$ to the erosion has to be flux dependent. A flux interpolation is done with the help of Roth's formula (mainly the thermal part $\left.\left(Y_{\text {therm }}\left(1+D Y_{\text {dam }}\right)\right)\right)$. 
The erosion yield given by the Mech model is correct only for a flux of $10^{18} m^{-2} s^{-1}$ (this is kept as the reference level flux $\Phi_{0}$ ). It has been observed that with increasing flux, the temperature at which the yield is maximum $\left(T_{\max }(E, \Phi)\right)$ and the absolute value of the yield $\left(Y_{\max }(E, \Phi)\right)$ increases. It is assumed that $T_{\max }(E, \Phi)$ predicted by the Roth model is correct (although $Y_{\max }(E, \Phi)$ is not). Then in order to calculate the flux dependent erosion yield for a given flux $\Phi$ and energy E, one proceeds as follows:

(1) First the $\Delta T$ from Roth's formula (see Fig. 8) is computed

$$
\Delta T=T_{\max }(E, \Phi)-T_{\max }\left(E, \Phi_{0}\right)
$$

(2) then the erosion yield from the Mech model at $T-\Delta T, \Phi_{0}$ is calculated

$$
Y_{T \_M e c h}\left(E, T-\Delta T, \Phi_{0}\right)
$$

(3) the corrected flux dependent erosion yield is given by

$$
Y_{T c o r}(E, T, \Phi)=Y_{T_{-} \text {Mech }}\left(E, T-\Delta T, \Phi_{0}\right) \cdot \frac{Y_{\text {Roth }}(E, T, \Phi)}{Y_{\text {Roth }}\left(E, T, \Phi_{0}\right)}
$$

If the sample is composed of $n$ layers, then the total flux dependent thermal erosion yield is

$$
Y_{T \_S D T r i m}(E, T, \Phi)=\sum_{1}^{n} \frac{Y_{T c o r}(E, T, \Phi)}{s p^{3}+s p_{C H}^{x}} \cdot e^{(-x / \lambda)}
$$


Finally the total erosion yield of carbon is:

$$
Y=Y_{\text {physical }}(E)+Y_{0 \_S D T r i m}\left(E, T_{0}\right)+Y_{T_{-} S D T r i m}(E, T, \Phi)
$$

Having developed the new module SDTrimSP, in the following part of this section the general algorithm in the simulation and its technical implementation is presented.

A pre-calculated number of energetic particles, depending on the fluence, are incident on the target. The target is divided into 1D layers and during each fluence step the incident particles initiate a collision cascade in the target. At the end of each fluence step sputtering yield (chemical and physical), scattering coefficient, and implantation is calculated and then the sample is updated accordingly.

Depending upon the incident particle and target combination it is checked after each collision whether some thermal chemical reactions are possible or not. If yes, then the chemistry module is used to implement the reactions. Then the depth dependent out-diffusion probability of the reactions products is also calculated and this is used to calculate the thermal part of the erosion yield.

During the cascade the number of broken bonds is calculated for each layer. During the collision with the target atoms the incident particles loose their energy and at the end of the collision cascade they get implanted into the target. The number of the implanted hydrogen atoms are calculated for each layer. At the end of the fluence step the total number of implanted hydrogen atoms and bond breaking are used for the calculation of the kinetic part of the erosion yield.

As explained in section (2.1) the maximum amount of bonded hydrogen has an upper limit of $1 / 3$. At the end of each fluence step the relative concentration of the bonded hydrogen is calculated and if it exceeds the upper limit the surplus hydrogen is treated as freely moving. This hydrogen can then diffuse through the target and leave the system. The corresponding interchange among the different carbon hybridization states is also done simultaneously.

The surface binding energy (SBE) for the pure carbon target (mainly $s p^{2}$ centers) is $7.37 \mathrm{eV}$ [14]. When this sample is bombarded with $A r^{+}$then $s p^{2}$ as well as $s p^{3}$ carbon centers are present and both of them should have the same SBE of $7.37 \mathrm{eV}$. In case of $H \rightarrow \mathrm{a}-\mathrm{H}$ :C the SBE of $s p^{3}$ carbon centers changes due to the presence of hydrogen. By fitting the experimental data for physical sputtering of $\mathrm{H}$ by $\mathrm{C}$ at $300 \mathrm{~K}$, a SBE of $4.50 \mathrm{eV}$ for $s p^{3}$ and $s p_{H}^{3}$ gives the best results (see Fig. 9). It must be noted that at $300 \mathrm{~K}$ the concentration of $s p^{2}$ carbon centers is negligible. At higher temperatures the SBE of $s p_{H}^{3}$ 
remains $4.50 \mathrm{eV}$ where as for $s p^{3}$ it depends on the ratio of $s p_{H}^{3}:\left(s p^{3}+s p_{H}^{3}\right)$. For example if one start with a pure carbon target, as the system evolves the relative concentrations of $s p^{2}, s p^{3}$ and $s p_{H}^{3}$ changes and accordingly the SBE of $s p^{3}$ also varies.

Finally all the counters for the bond breaking and hydrogen implantation in each layer are reset to zero.

\section{Further validation}

Using SDTrimSP the calculations were performed to study the two experiments namely $H \rightarrow C$ and $A r \mid H \rightarrow$ a-C:H. Temperature dependence of erosion yield for these two cases are shown in Fig. 2 and 3. It can be observed that the simulation results obtained from the new SDTrimSP version follows very well the trends of both the experiments.

Fig. 4 shows the temperature dependence of the erosion yield for $200 \mathrm{eV}$ and Fig. 5 shows the energy dependence of the erosion yield at $700 \mathrm{~K}$. The simulation results by SDTrimSP matches very well the energy and temperature dependence of the chemical erosion yield.

The flux dependence of erosion yield obtained from SDTrimSP is in good agreement with the experimental data (Fig. 6 and 7). The analytical models available do not predict the flux, temperature and energy dependence of erosion very well. But the combination of important elements from different models leads to good agreement with the experiments.

In Fig. 2 the chemical sputtering yield and in Fig. 9 the total sputtering yield (including physical sputtering as well) for different energies is presented. From Fig. 2 it is clear that the chemical erosion yield is negligible at higher energies $(>1 \mathrm{keV})$ so the sputtering yield at higher energies in Fig. 2 is mainly due to the physical sputtering. The very good agreement between the experiments and the simulation results from SDTrimSP shows that the choice of SBE is reasonable.

The aim of the present work was to develop a model which can reproduce the experimental results of the flux, temperature and energy dependence of the chemical erosion.

Different experimental data and theoretical models were taken and then they were used to parameterize different physics aspects of the problem. The fitted values were used to develop the new module of SDTrimSP presented in this paper and then the new SDTrimSP version was validated against most of the 
experimental data available.

A first illustration of the quality of the new model is shown in Fig. (9). Experimental data from $[6,3]$ measured at room temperature for two different flux densities show no influence of the flux density on the measured total steady state sputtering yield. The new module is able to reproduce both the values and the dependence on incidence energy of hydrogen quite well.

Also, no effect is observed within SDTrimSP for the two different flux densities. The experimental data at $800 \mathrm{~K}$ for a fixed flux density $[6,13]$ show a quite different dependence of the total steady state sputtering yield with respect to the energy of the impinging hydrogen compared to the data at $300 \mathrm{~K}$. The model is able to reproduce this as well.

Further checks of the model are done (see Fig. 10) for the temperature dependence of the chemical erosion yield $([6,13])$ for a fixed energy and flux density of hydrogen. The onset and maximum is reasonable well reproduced by the model.

Fig. 11 shows the flux dependence of the chemical erosion yield at 750 and $850 \mathrm{~K}$. The model does not agree perfectly in this case, but still the tendencies are reproduced. The sensitivity of this particular comparison is obvious, taking into account that the same calculated data for $700 \mathrm{~K}$ fit nearly perfect the experimental one, whereas in the case of $800 \mathrm{~K}$ one observes larger deviations. Here, the possible large error bars of the experimental data should be considered. In fact, the new model reproduces at least the trends quite well.

Application of the new model is also able to deliver information about the system, which is nearly impossible to obtain experimentally. As an example, Fig. 12 shows the development of the depth profiles of hydrogen, $s p^{2}$ and $s p^{3}$ for different fluences for an energy of $200 \mathrm{eV}$ of incident hydrogen atoms. After a fluence of $1 \cdot 10^{20} \mathrm{~m}^{-2}$ nearly all $s p^{2}$ is converted into $s p^{3}$ within the penetration depth of hydrogen. With increased fluence, more and more implanted hydrogen is bonded to an open bond of carbon and as a consequence more and more $s p^{3}$ is transferred into $s p_{H}^{3}$ and a $\mathrm{H}: \mathrm{C}$ layer is formed. The formation of a saturation layer starts at about $5 \cdot 10^{20} \mathrm{~m}^{-2}$ until it equilibrates over the full depth range. Implanted hydrogen, which does not form bonds with carbon, is considered to experience induced diffusion into the direction of the surface [1] and can leave the system. In steady-state, a typical H:C layer is developed with a $\mathrm{H} /(\mathrm{H}+\mathrm{C})$ ratio of $1: 3$. During this process, both physical and chemical sputtering and scattering of hydrogen occur. Fig. 13 shows the development of the physical and chemical sputtering yields. For a fluence of $5 \cdot 10^{20} \mathrm{~m}^{-2}$ saturation of hydrogen is reached. With further increase of the fluence the additional hydrogen diffuses through the surface and the saturated layer penetrated deeper in the sample but at these depths the escape 
probability of the hydrocarbons is very low and therefore we observe a quasi steady-state where the erosion yield are practically constant. The start of the saturation is clearly visible in the sputtering yields (at fluence of $6 \cdot 10^{20} \mathrm{~m}^{-2}$ ), especially in the chemical one.

Fig. 14 shows the depth profile of the point of origin for hydrocarbon molecules at different energies. At lower energy of $0.01 \mathrm{keV}$ total sputtering is dominated by the chemical erosion and contribution from the physical sputtering is negligible. In general, the process of chemical sputtering is maximum close to the surface and decays rapidly with increasing depth. In case of physical sputtering significant contribution comes even from deeper layers.

\section{Summary and conclusion}

The new version of the program SDTrimSP calculates the physical and chemical sputtering of carbon due to (i) $\mathrm{H}$ or (ii) $\mathrm{Ar}^{+}$and $\mathrm{H}$. The results matches very well with the experiments for different parameter range of energy, temperature and flux. The simulations also provides information about the depth profile of various species for different incident fluences and the dynamics of the erosion process. In the present work no attempt is made to consider radiation damage, for example, the creation of voids, clustering of implanted ions or inhomogeneity in the density of the target. 


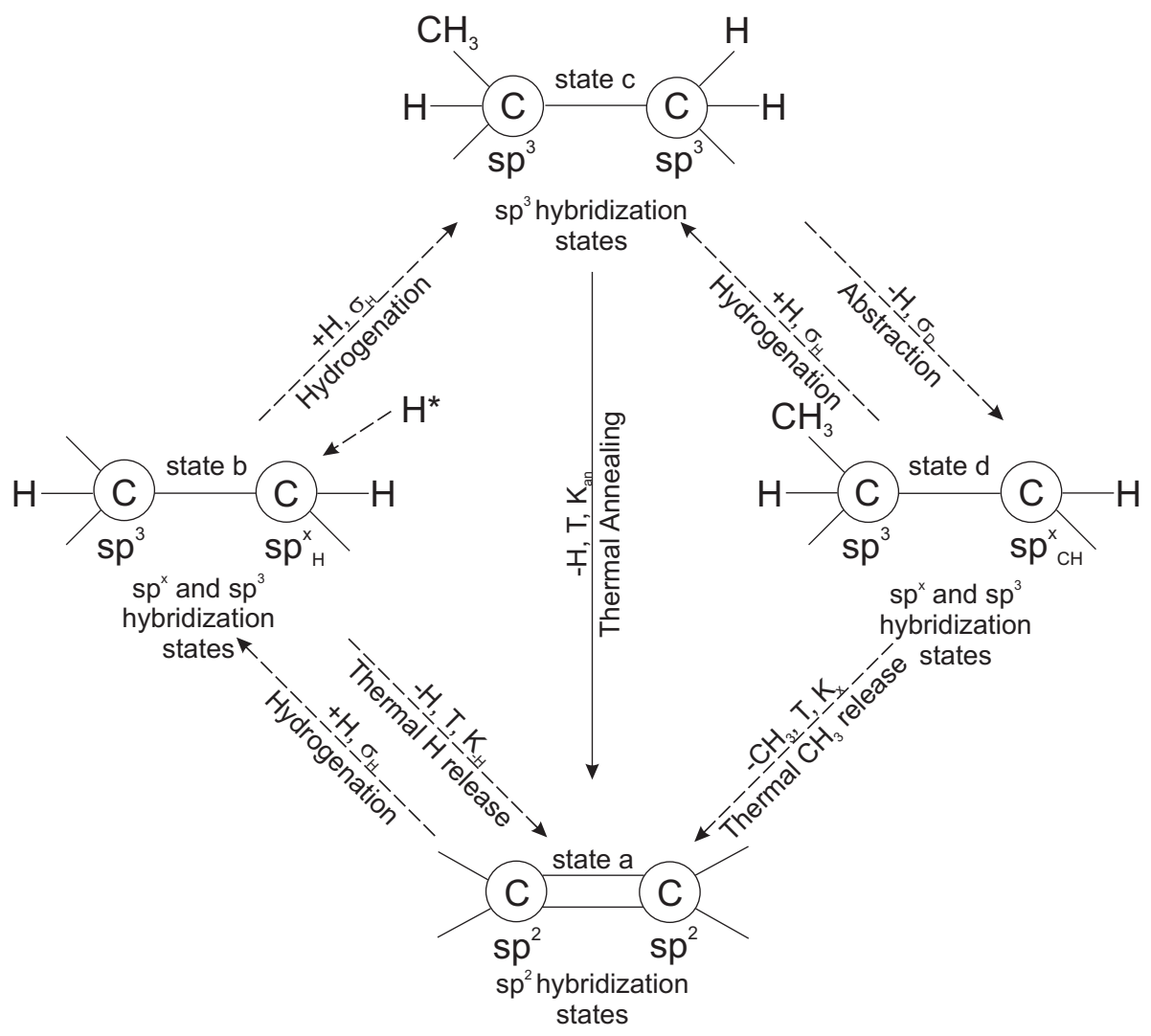

Fig. 1. Schematic diagram of the reactions leading to methane production due to atomic hydrogen impact on amorphous hydrogenated carbon films, as proposed by Küppers and co-workers $[8,5,3]$.

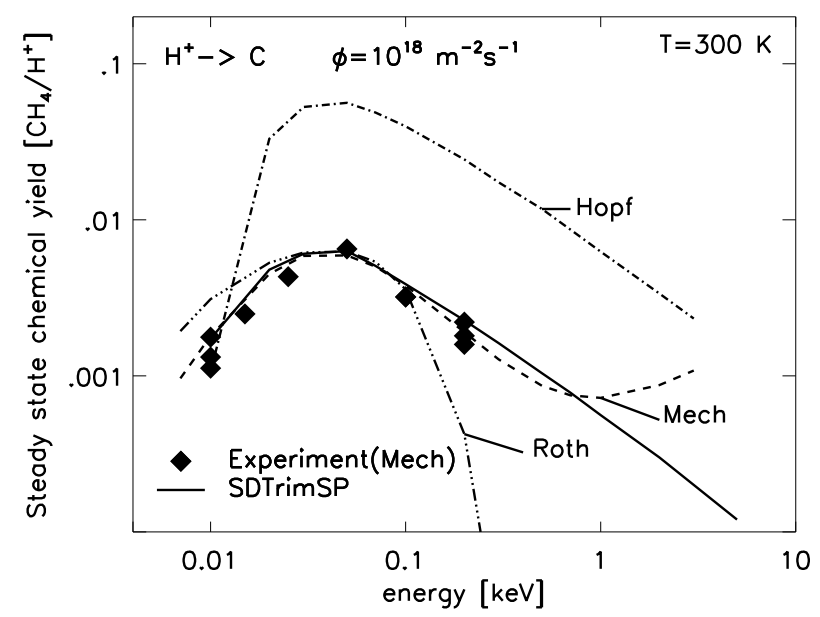

Fig. 2. Calculated room temperature erosion yields of $\mathrm{H}$ on a $\mathrm{C}$ target at normal incidence as a function of the incident energy of $\mathrm{H}$ compared with experimental data $[3]$. 


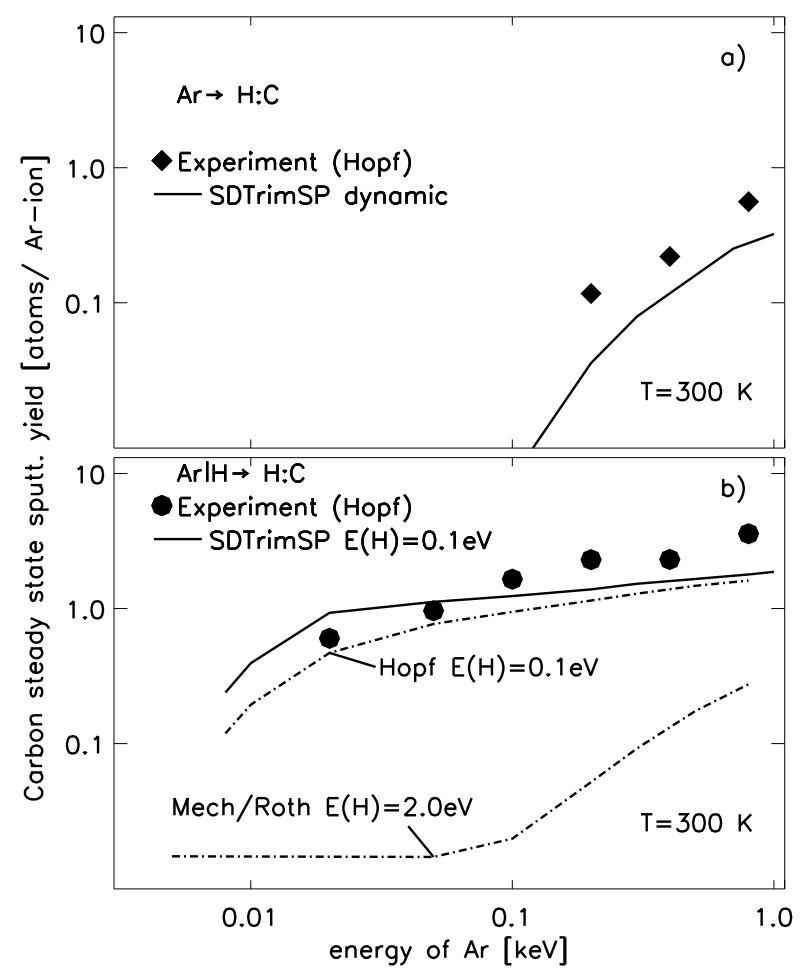

Fig. 3. Calculated steady state sputtering yields versus the incident energy of Ar on a $\mathrm{H}: \mathrm{C}$ target at normal incidence a) without and b) with thermal $\mathrm{H}$ flux. The results are compared with experimental data [7]. $E(H)$ is the energy of thermal hydrogen used in the different models.

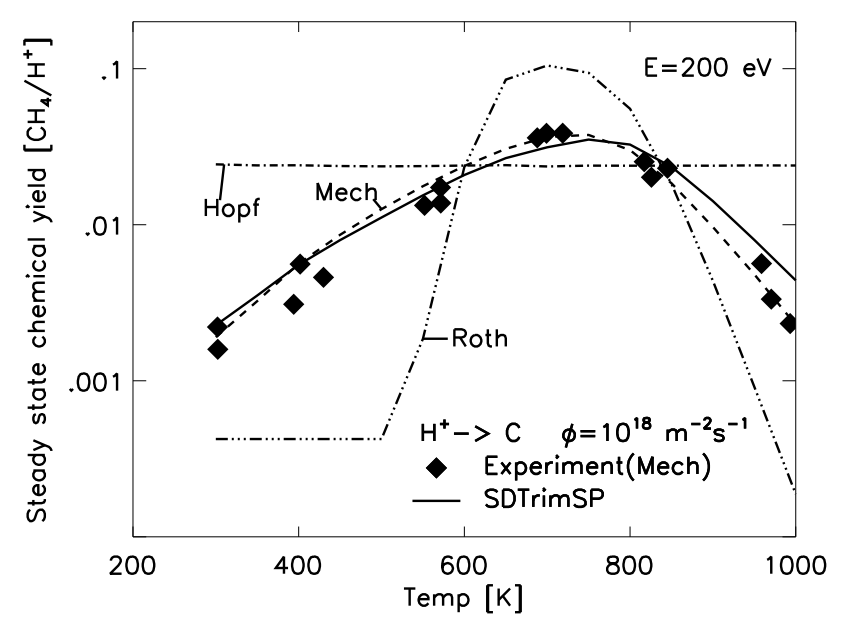

Fig. 4. Calculated steady state methane yields of $\mathrm{H}$ on a $\mathrm{C}$ target at normal incidence as a function of target temperature compared with experimental data [3]. 


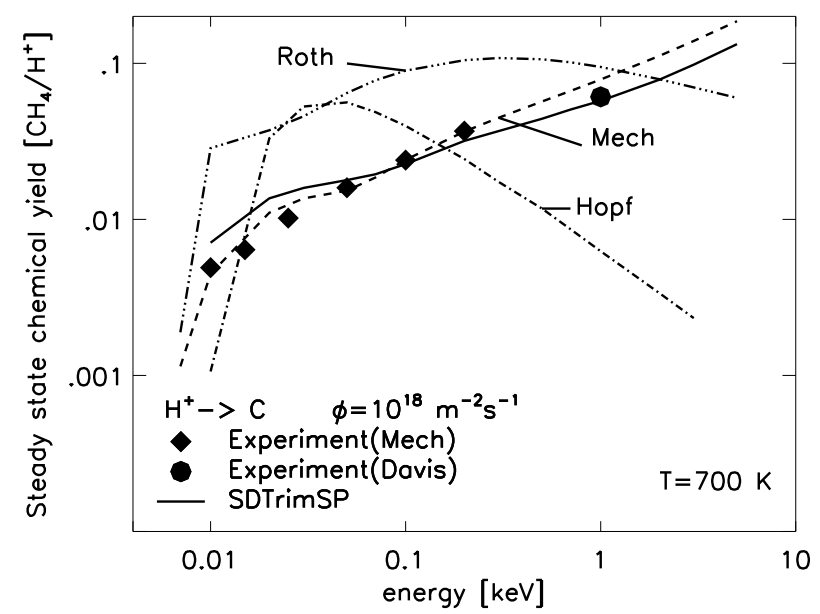

Fig. 5. Calculated steady state methane yields of $\mathrm{H}$ on a $\mathrm{C}$ target at normal incidence as a function of the ion energy of $\mathrm{H}$ compared with experimental data [3] at $700 \mathrm{~K}$. 


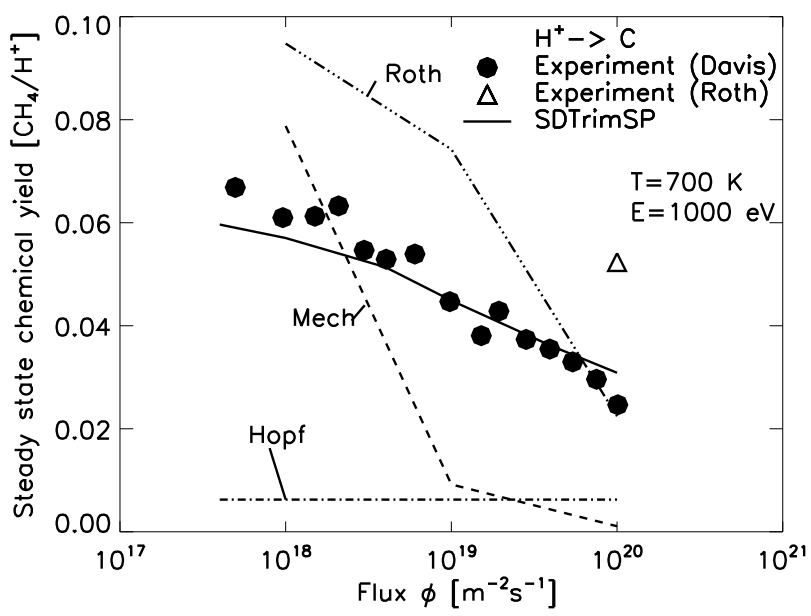

Fig. 6. Calculated steady state methane yields $\mathrm{T}=700 \mathrm{~K}$ of $\mathrm{H}$ on a $\mathrm{C}$ target at normal incidence as a function of hydrogen flux compared with experimental data [13].

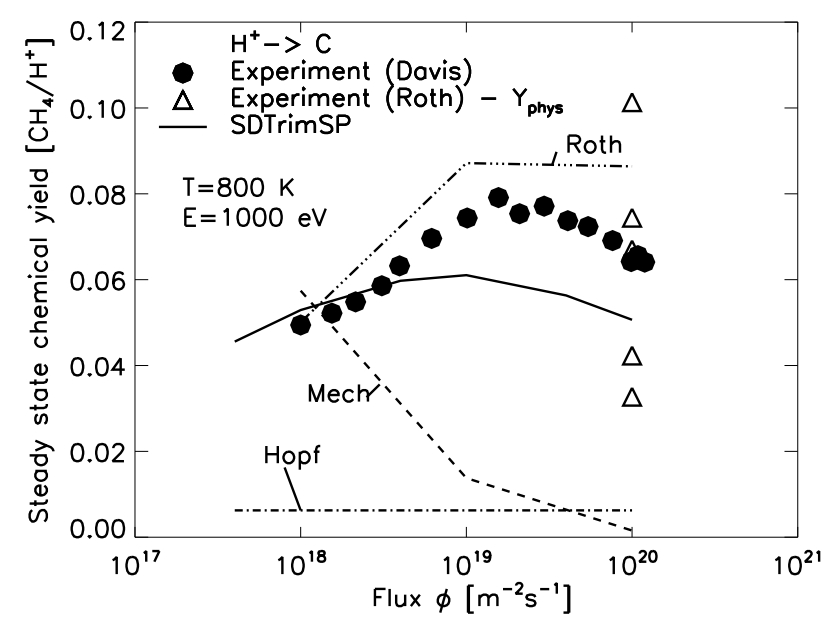

Fig. 7. Calculated steady state methane yields $\mathrm{T}=800 \mathrm{~K}$ of $\mathrm{H}$ on a $\mathrm{C}$ target at normal incidence as a function of hydrogen flux compared with experimental data [13]. 


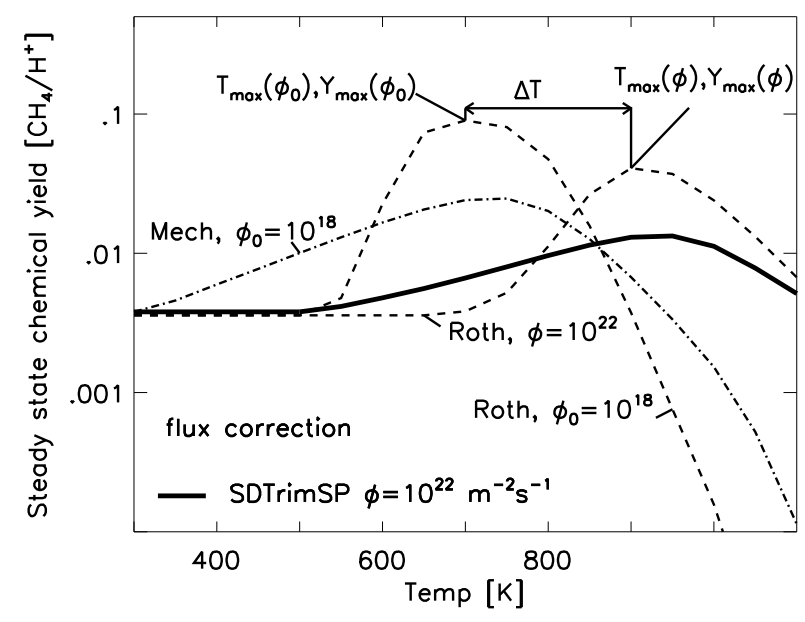

Fig. 8. Correction of thermal erosion yield of Mech [3] at a $\mathrm{H}$ flux $\Phi=10^{18} \mathrm{~m}^{-2} \mathrm{~s}^{-1}$ to get values at $\Phi=10^{22} \mathrm{~m}^{-2} \mathrm{~s}^{-1}$ (SDTrimSP) using the analytical formula by Roth [6].

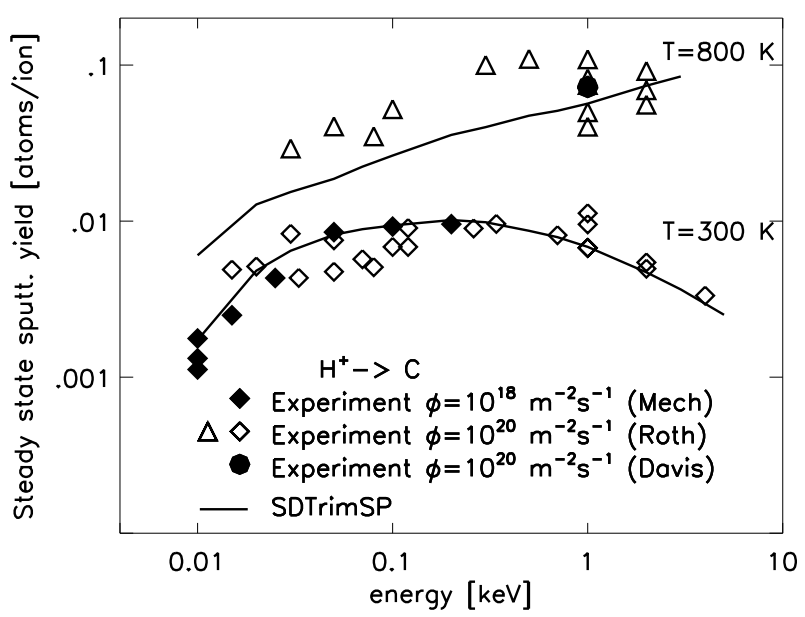

Fig. 9. Calculated steady state sputtering yields $\mathrm{T}=300 \mathrm{~K}$ and $\mathrm{T}=800 \mathrm{~K}$ of $\mathrm{H}$ on a $\mathrm{C}$ target at normal incidence as a function of energy compared with experimental data $[3,6]$. 


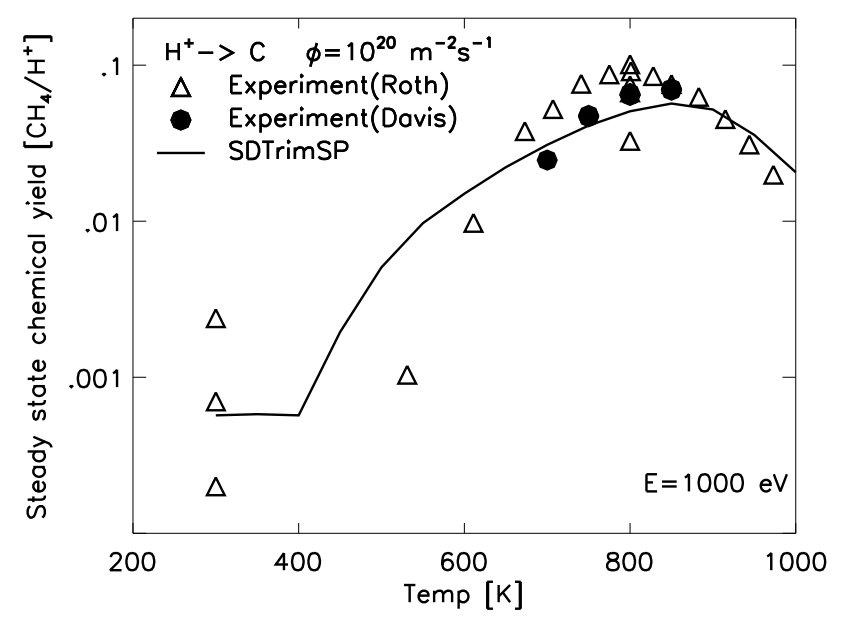

Fig. 10. Calculated steady state methane yields at $1 \mathrm{keV}$ of $\mathrm{H}$ on a $\mathrm{C}$ target at normal incidence as a function of target temperature compared with experimental data $[6,13]$.

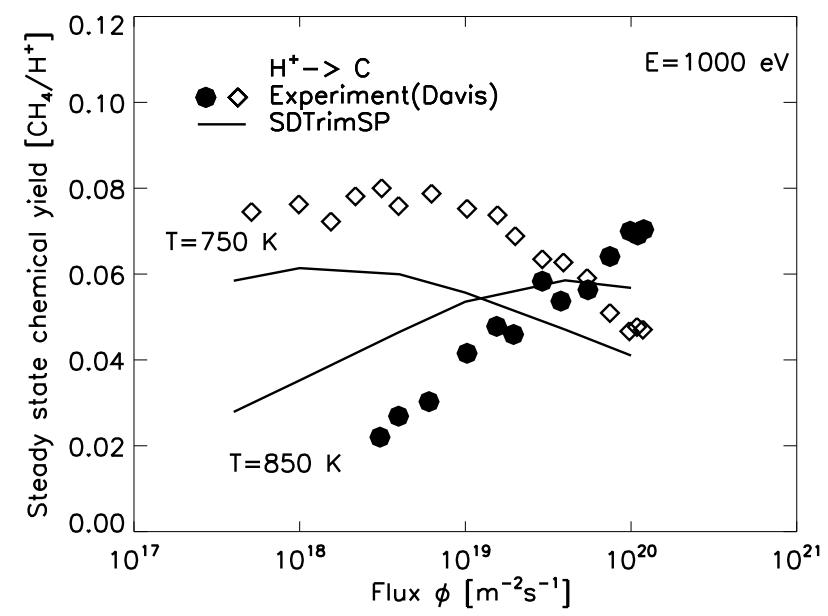

Fig. 11. Calculated steady state methane yields for $750 \mathrm{~K}$ and $850 \mathrm{~K}$ versus the flux of $\mathrm{H}$ on a $\mathrm{C}$ target at normal incidence compared with experimental data [13]. 

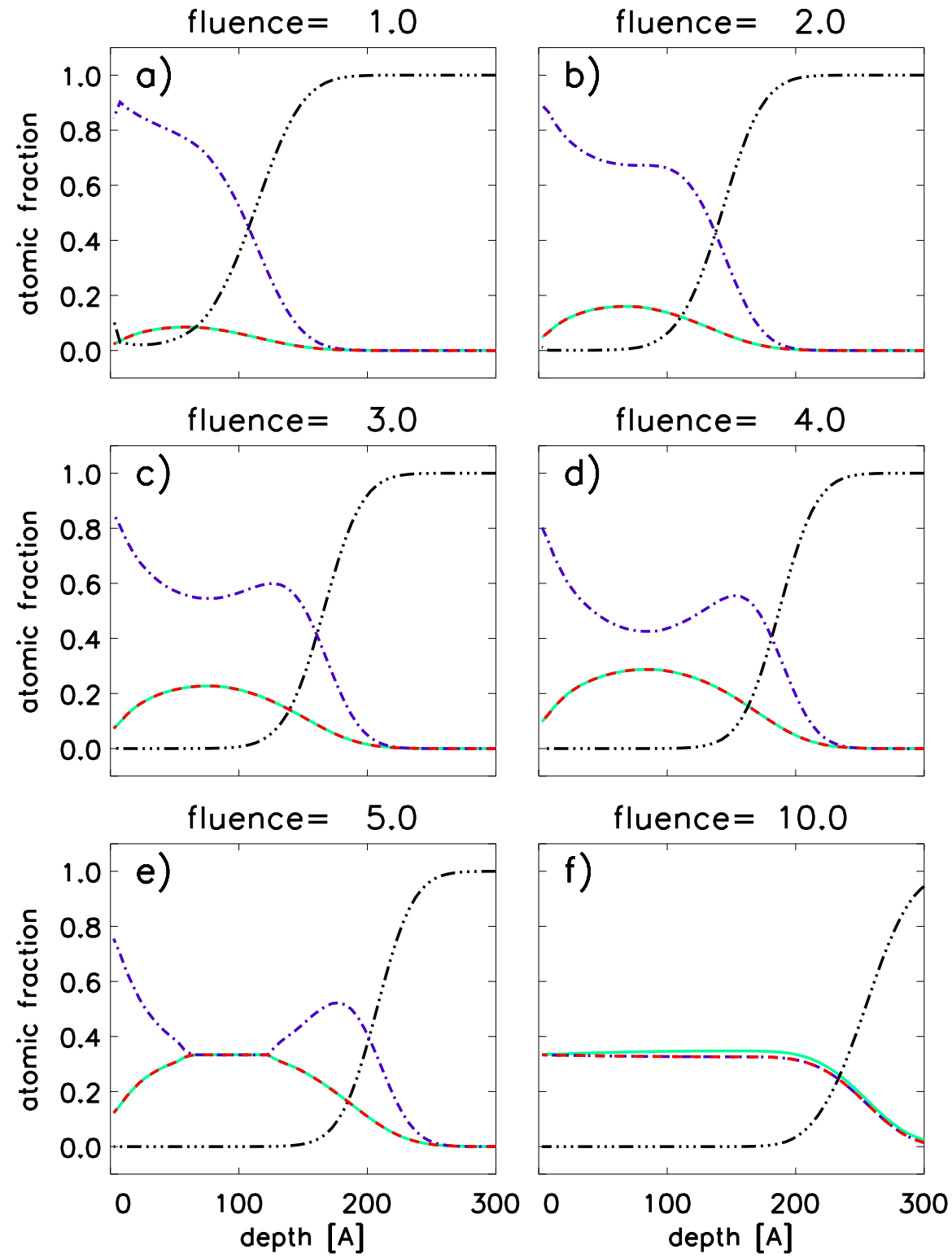

$-\mathrm{H} \quad$-...sp2

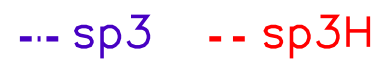

Fig. 12. Development of the profile of $H, s p^{2}, s p^{3}$ and $s p_{H}^{3}$ for $\mathrm{H} \rightarrow \mathrm{a}-\mathrm{C}: \mathrm{H}$ with an energy of $200 \mathrm{eV}$ at different fluences ranging from $1 \cdot 10^{20}-10 \cdot 10^{20} \mathrm{~m}^{-2}$. 


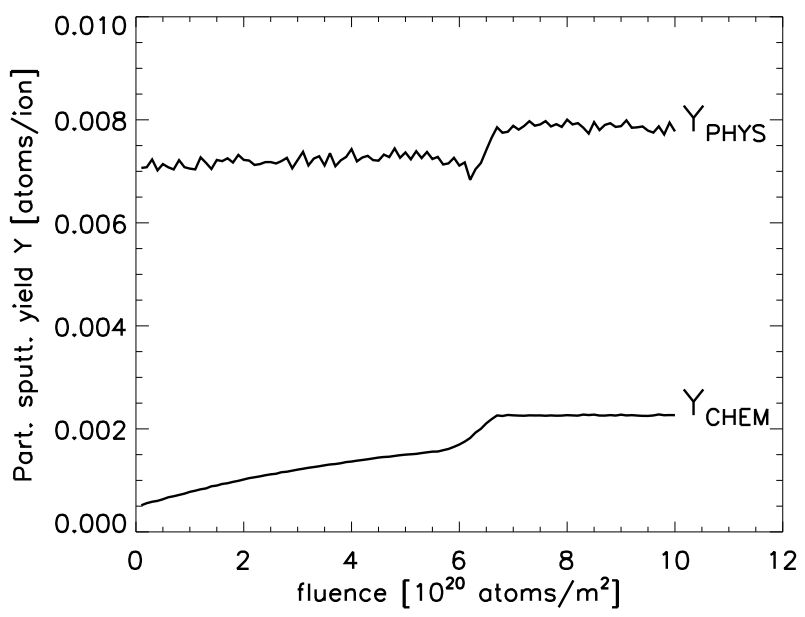

Fig. 13. Development of physical and chemical sputtering yield of carbon for $\mathrm{H} \rightarrow$ $\mathrm{a}-\mathrm{C}: \mathrm{H}$ target with energy of $200 \mathrm{eV}$.

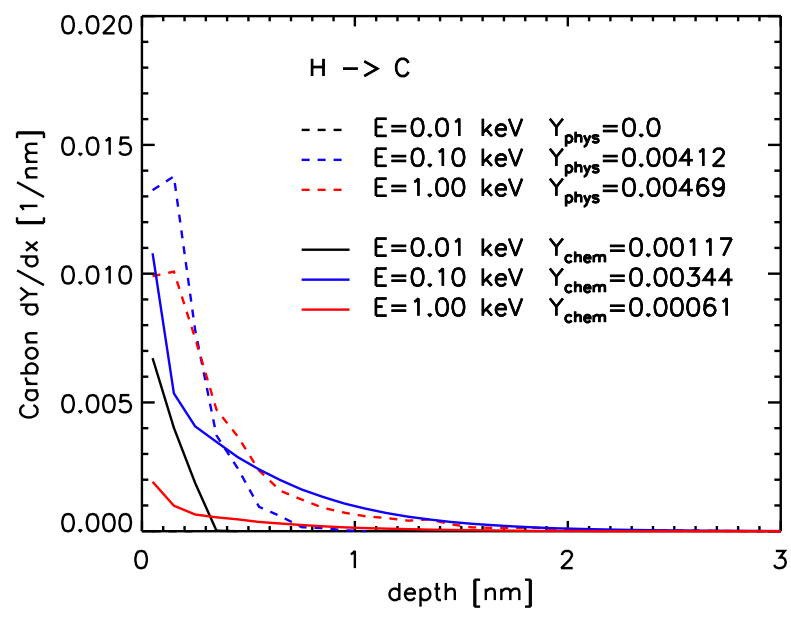

Fig. 14. Depth profile of the Point of formation for hydrocarbon molecules. 


\section{References}

[1] A. Mutzke and W. Eckstein. Nucl. Instr. amd Meth. B, 266:872-876, (2008).

[2] W. Eckstein, R. Dohmen, and A. Mutzke. SDTrimSP: A Monte-Carlo Code for Calculating Collision Phenomena in Randomized Targets. IPP, Report, (12/3), 2007.

[3] B. V. Mech, A. A. Haasz, and J. W. Davis. J. Appl. Phys., 84(3):1655-1669, (1998).

[4] J. Küppers. Surface Science Reports, 22:249-321, (1995).

[5] M. Wittmann and J. Küppers. J. Nucl. Mater., 227:186-194, (1996).

[6] J. Roth and C. Garcia-Rosales. Nucl. Fusion, 36:1647-1659, (1996).

[7] C. Hopf, A. von Keudell, and W. Jacob. J. Appl. Phys., 94:2376, (2003).

[8] A. Horn, A. Schenk, J. Biener, B. Winter, C. Lutterloh, M. Wittman, and J. Küppers. Chem. Phys. Lett., 231(2-3):193-198, (1994).

[9] E. Salonen, K. Nordlund, J. Tarus, T. Ahlgren, J. Keinonen, and C. H. Wu. Phys. Rev. B, 60:14005-14008, (1999).

[10] E. Salonen, K. Nordlund, J. Keinonen, and C. H. Wu. J. Nucl. Mater., 290293:144-147, (2001).

[11] A. Rai, R. Schneider, M. Warrier, P. Roubin, and C. Martin. J. Nucl. Mater., 390-391:655-658, (2009).

[12] A. Rai, R. Schneider, and M. Warrier. J. Nucl. Mater, 374:304-312, (2008).

[13] J. W. Davis, A. A. Haasz, and P. C. Stangby. J. Nucl. Mater., 145-147:417-420, (1987).

[14] W. Eckstein. Computer Simulation of Ion-Solid Interactions. Springer Series in Material Science, Berlin, Heidelberg, 1991. 


\section{List of Figures}

1 Schematic diagram of the reactions leading to methane production due to atomic hydrogen impact on amorphous hydrogenated carbon films, as proposed by Küppers and co-workers $[8,5,3]$.

2 Calculated room temperature erosion yields of $\mathrm{H}$ on a $\mathrm{C}$ target at normal incidence as a function of the incident energy of $\mathrm{H}$ compared with experimental data [3].

3 Calculated steady state sputtering yields versus the incident energy of $\mathrm{Ar}$ on a $\mathrm{H}$ : $\mathrm{C}$ target at normal incidence a) without and b) with thermal $\mathrm{H}$ flux. The results are compared with experimental data [7]. $E(H)$ is the energy of thermal hydrogen used in the different models.

4 Calculated steady state methane yields of $\mathrm{H}$ on a $\mathrm{C}$ target at normal incidence as a function of target temperature compared with experimental data [3].

5 Calculated steady state methane yields of $\mathrm{H}$ on a $\mathrm{C}$ target at normal incidence as a function of the ion energy of $\mathrm{H}$ compared with experimental data [3] at $700 \mathrm{~K}$.

6 Calculated steady state methane yields $\mathrm{T}=700 \mathrm{~K}$ of $\mathrm{H}$ on a $\mathrm{C}$ target at normal incidence as a function of hydrogen flux compared with experimental data [13].

7 Calculated steady state methane yields $\mathrm{T}=800 \mathrm{~K}$ of $\mathrm{H}$ on a $\mathrm{C}$ target at normal incidence as a function of hydrogen flux compared with experimental data [13].

8 Correction of thermal erosion yield of Mech [3] at a $\mathrm{H}$ flux $\Phi=10^{18} \mathrm{~m}^{-2} \mathrm{~s}^{-1}$ to get values at $\Phi=10^{22} \mathrm{~m}^{-2} \mathrm{~s}^{-1}$ (SDTrimSP) using the analytical formula by Roth [6].

9 Calculated steady state sputtering yields $\mathrm{T}=300 \mathrm{~K}$ and $\mathrm{T}=800$ $\mathrm{K}$ of $\mathrm{H}$ on a $\mathrm{C}$ target at normal incidence as a function of energy compared with experimental data $[3,6]$.

10 Calculated steady state methane yields at $1 \mathrm{keV}$ of $\mathrm{H}$ on a $\mathrm{C}$ target at normal incidence as a function of target temperature compared with experimental data $[6,13]$. 
11 Calculated steady state methane yields for $750 \mathrm{~K}$ and 850 $\mathrm{K}$ versus the flux of $\mathrm{H}$ on a $\mathrm{C}$ target at normal incidence compared with experimental data [13].

12 Development of the profile of $H, s p^{2}, s p^{3}$ and $s p_{H}^{3}$ for $\mathrm{H} \rightarrow$ a-C:H with an energy of $200 \mathrm{eV}$ at different fluences ranging from $1 \cdot 10^{20}-10 \cdot 10^{20} m^{-2}$.

13 Development of physical and chemical sputtering yield of carbon for $\mathrm{H} \rightarrow$ a- $\mathrm{C}: \mathrm{H}$ target with energy of $200 \mathrm{eV}$.

14 Depth profile of the Point of formation for hydrocarbon molecules. 


\section{Appendices}

\section{A Model by Roth}

The total erosion yield of carbon under hydrogen bombardment, $Y_{t o t}$, consists of three components, the physical sputtering yield $\left(Y_{\text {phys }}\right)$, the thermal erosion yield $\left(Y_{\text {therm }}\right)$, enhanced by radiation damage $\left(Y_{\text {dam }}\right)$ and the sputtering of radicals $\left(Y_{\text {surf }}\right)$.

$$
Y_{\text {tot }}=Y_{\text {phys }}+Y_{\text {therm }}\left(1+D \cdot Y_{\text {dam }}\right)+Y_{\text {surf }}
$$

For an ion energy $E_{0}$

$$
\begin{aligned}
& Y_{\text {phys }}=Q \cdot S\left(1-E^{2 / 3}\right)(1-E)^{2} \quad \text { with } \quad E=E_{\text {th }} / E_{0} \\
& Y_{\text {dam }}=Q \cdot S\left(1-E^{2 / 3}\right)(1-E)^{2} \quad \text { with } \quad E=E_{\text {dam }} / E_{0} \\
& Y_{\text {des }}=Q \cdot S\left(1-E^{2 / 3}\right)(1-E)^{2} \quad \text { with } \quad E=E_{\text {des }} / E_{0} \\
& Y_{\text {surf }}=c^{s p 3} \frac{Y_{\text {des }}}{1+\exp \left(\frac{E_{0}-65}{40}\right)}
\end{aligned}
$$

The thermal erosion yield at an ion flux $\Phi$, can be obtained by

$$
Y_{\text {therm }}=c^{s p 3} \frac{0.033 \cdot \exp \left(\frac{-E_{\text {therm }}}{k T}\right)}{2 \cdot 10^{-32} \cdot \Phi+\exp \left(\frac{-E_{\text {therm }}}{k T}\right)}
$$

where D, Q, $E_{t f}$ (Thomas-Fermi energy for the ion target interaction), $E_{t h}$, $E_{\text {therm }}, E_{\text {dam }}$, and $E_{\text {des }}$ are parameters depending on the hydrogen isotope (given in table 1). $c^{s p^{3}}$ is the concentration of the $s p^{3}$ carbon centers and $\mathrm{S}$ is the nuclear stopping power approximated by

$$
\begin{aligned}
& S=\frac{0.5 \cdot \ln (1+1.2288 \cdot E)}{E+0.1728 \sqrt{E}+0.008 \cdot E^{0.1504}} \quad \text { with } E=E_{0} / E_{t f} \\
& c^{s p 3}=\frac{c \cdot\left(2 \cdot 10^{-32} \cdot \Phi+\exp (E)\right)}{2 \cdot 10^{-32} \cdot \Phi+\left[1+\frac{2 \cdot 10^{29}}{\Phi} \cdot \exp \left(\frac{-E_{r e l}}{k T}\right)\right] \cdot \exp \left(\frac{-E_{\text {therm }}}{k T}\right)} \\
& c=\frac{1}{\left(1+3 \cdot 10^{7} \cdot \exp \left(\frac{-1.4}{k T}\right)\right)} .
\end{aligned}
$$


For high ion fluxes the correction term c is replaced by

$$
c=\frac{1}{\left(1+3 \cdot 10^{-23} \cdot \Phi\right)}
$$

where the flux $\Phi$ is given in ions $/ \mathrm{m}^{2} / \mathrm{s}$. The parameters used in Eqs. A.1 A.10 are provided in Table 1.

Table A.1

Parameters for chemical erosion

\begin{tabular}{|r|rrr|}
\hline Parameter & Hydrogen & Deuterium & Tritium \\
\hline$E_{t f}$ & $415 \mathrm{eV}$ & $447 \mathrm{eV}$ & $479 \mathrm{eV}$ \\
$\mathrm{Q}$ & 0.035 & 0.1 & 0.12 \\
$E_{t h}$ & $31 \mathrm{eV}$ & $27 \mathrm{eV}$ & $29 \mathrm{eV}$ \\
$E_{\text {dam }}$ & $15 \mathrm{eV}$ & $15 \mathrm{eV}$ & $15 \mathrm{eV}$ \\
$E_{\text {des }}$ & $2 \mathrm{eV}$ & $2 \mathrm{eV}$ & $2 \mathrm{eV}$ \\
$E_{\text {rel }}$ & $1.8 \mathrm{eV}$ for pure carbon & & \\
& $1.5 \mathrm{eV}$ for Si,Ti,W doped carbon & & \\
& $1.2 \mathrm{eV}$ for B doped carbon & & \\
$E_{\text {therm }}$ & 1.7 & & \\
$\mathrm{D}$ & 250 & 125 & 83 \\
\hline
\end{tabular}

\section{B Extended Küppers model by Mech}

In this subsection we present the detailed rate equations for chemical erosion obtained by Mech et al.. The rate equation for every process is written with the bracketed quantities as concentrations and $\Phi$ as $\mathrm{H}$ atom flux. The system can be described by the following set of differential equations:

(1) Transformation from graphitic $s p^{2}$ to $s p_{H}^{x}$ with a neighboring $s p^{3}$ carbon center (state a to state b) can occur either by the hydrogenation of a $s p^{2}$ carbon center by a thermal hydrogen or an incoming energetic hydrogen ion can break the double bond between two $s p^{2}$ carbon centers leading to bond arrangement producing state $\mathrm{b}$. The corresponding rate equations are

$$
\begin{aligned}
& -\frac{d\left[s p^{2}\right]}{d t}=\left[s p^{2}\right] \sigma_{H} \Phi \\
& -\frac{d\left[s p^{2}\right]}{d t}=\left[s p^{2}\right] \sigma_{E} \Phi
\end{aligned}
$$


Here $\sigma_{H}\left(=4.5 \times 10^{-20} \mathrm{~m}^{2}\right)$ is the hydrogenation cross-section and $\sigma_{E}$ is the fitting parameter giving effective cross-section for the bond breaking and rearrangement of one of the graphitic $s p^{2}$ carbon by an incident energetic $\mathrm{H}$ ion. For hydrogen ions with energy $\mathrm{U}$ (in $\mathrm{eV}$ ), mass $\mathrm{M}$ (in amu) and $\Phi=10^{18} \mathrm{~m}^{-2} \mathrm{~s}^{-1}$,

$$
\sigma_{E}=9.9 \times 10^{-17} \sqrt{M} \ln \left(1+0.00334(U-5)^{2}\right), U>5 e V
$$

At higher temperatures split-off of this hydrogen (dehydrogenation) can return the two carbon centers to their original state a.

$$
-\frac{d\left[s p_{H}^{x}\right]}{d t}=\left[s p_{H}^{x}\right] k_{-H} \exp \left(\frac{-E_{-H}}{k T}\right)
$$

where $k_{-H}\left(=10^{13} s^{-1}\right)$ is the frequency factor and $E_{-H}$ is the activation energy for this process having a normal distribution with mean value of $1.73 \mathrm{eV}$ and a width of $0.52 \mathrm{eV}$.

(2) Repetitive application of the hydrogenation to $s p_{H}^{x}$ leads to $s p^{3}$, some fraction of which contains a methyl group attached (state b to state $\mathrm{c}$ ).

$$
-\frac{d\left[s p_{H}^{x}\right]}{d t}=\left[s p_{H}^{x}\right] \sigma_{H} \Phi
$$

At low impact energies, kinetic ejection of the loosely bonded hydrocarbons attached as ligands to $s p^{3}$ carbon (state c) can take place. Since the surface concentration of these $s p^{3}$ carbon centers is high at low temperature this effect is dominant at low impact energies and lower temperatures. The rate equation for this process is:

$$
-\frac{d\left[s p^{3}\right]}{d t}=\left[s p^{3}\right] \sigma_{i} \Phi
$$

Here $\sigma_{i}$ is the effective cross-section for the kinetic ejection of a methyl group. This is also a fitting parameter given by

$$
\sigma_{i}=\frac{18.1 \times 10^{-23} \sqrt{M} \ln \left(1+0.0033(U-1)^{1.85}\right)}{1+0.0022(U-1)^{1.77}}, U>1 \mathrm{eV}
$$

(3) Abstraction of a singly bonded hydrogen can produce a carbon in the radical $s p_{C H}^{x}$ configuration with a neighboring methyl-containing carbon in the $s p^{3}$ hybridization state (state c to state $\mathrm{d}$ ).

$$
-\frac{d\left[s p^{3}\right]}{d t}=\left[s p^{3}\right] k_{D} \exp \left(\frac{-E_{D}}{k T}\right)
$$

$E_{D}$ is the activation energy for the dehydrogenation process and it is also normally distributed about $0.26 \mathrm{eV}$ with a width of $0.14 \mathrm{eV} . k_{D}$ is 
the frequency pre-factor and given by the following fitted form

$$
k_{-D}=\left\{\begin{array}{l}
0.0068+2.5 \times 10^{-5} \cdot M \cdot U, U<45 \mathrm{eV} \\
0.0068+2.5 \times 10^{-5} \cdot M \cdot U+0.006 \\
. M \ln \left(1+0.002(U-45)^{1.8}\right), \quad U>45 \mathrm{eV} .
\end{array}\right.
$$

Hydrogenation can reverse the above reaction (state $d$ to state $\mathrm{c}$ ).

(4) For temperatures above $\sim 400 \mathrm{~K}$, the radical can de-excite by split-off of the neighboring methyl group and return the carbon atoms to graphitic $s p^{2}$ state (state d to state a).

$$
-\frac{d\left[s p_{C H}^{x}\right]}{d t}=\left[s p_{C H}^{x}\right] k_{x} \exp \left(\frac{-E_{x}}{k T}\right)
$$

where $k_{x}\left(=10^{13} s^{-1}\right)$ is the frequency factor and the normally distributed activation energy $E_{x}$ for thermal release of methyl group has a mean of $1.47 \mathrm{eV}$ and a width of $0.41 \mathrm{eV}$.

(5) For graphite temperatures in excess of $\sim 1100 \mathrm{~K}$ thermal annealing can return $s p^{3}$ carbon atoms to $s p^{2}$ state (state c to state a) via elimination of some of the ion-induced damage accumulated in the implantation zone.

$$
-\frac{d\left[s p^{3}\right]}{d t}=\left[s p^{3}\right] k_{a n} \exp \left(\frac{-E_{a n}}{k T}\right)
$$

where $k_{a n}\left(=10^{13} \mathrm{~s}^{-1}\right)$ is the frequency factor and $E_{a n}(=2.42 \mathrm{eV})$ is the activation energy for the annealing.

Thus, at low temperatures, exposure of the film to thermal hydrogen atoms results in the conversion of carbon from the $s p^{2}$ to the $s p^{3}$ hybridization state with some fraction of radical centers, determined by the ratio of hydrogenation and dehydrogenation. At higher temperatures (400-650 K) an equilibrium balance between the supply of $s p^{x}$ radical carbon centers from hydrogenation, or abstraction and their loss through thermal decomposition due to methyl or hydrogen split-off is established leading to measurable erosion rates. At even higher temperatures, however, the rapid decomposition of the radical states by $\mathrm{H}$-atom split-off prevents significant hydrogenation to $s p^{3}$ hybridization states. This, in turn, means there is little or no abstraction occurring, and so, there is no significant production of carbon in the radical carbon state. Thus, chemical erosion is suppressed at higher temperatures.

The methane erosion yield is composed of a kinetic part $Y_{0}$ and a thermal part $Y_{T}$. For given steady state concentration of $s p^{3}$ and $s p_{C H}^{x}$ and $\sigma_{i}$ the methane yield is: 


$$
\begin{aligned}
& Y_{\mathrm{CH}_{4}}=\quad Y_{0}\left(E, T_{0}\right) \quad+Y_{T}(E, T) \\
& Y_{C H_{4}}=\varrho \cdot \sigma_{i} \cdot\left[s p^{3}+s p_{C H}^{x}\right]+\varrho \cdot s p_{C H}^{x} \frac{E x}{\Phi} \\
& Y_{\mathrm{CH}_{4}}=\quad Y_{0 \mathrm{Mech}}+Y_{\text {TMech }}
\end{aligned}
$$

